\title{
VAGUENESS AND INTUITIONISTIC LOGIC
}

\section{Ian Rumfitt}

\begin{abstract}
In his essay “"Wang's Paradox", Crispin Wright proposed a solution to the Sorites Paradox (in particular, the form of it he calls the 'Paradox of Sharp Boundaries') that involves adopting intuitionistic logic when reasoning with vague predicates. He does not give a semantic theory which accounts for the validity of intuitionistic logic (and the invalidity of stronger logics) in that area. The present essay tentatively makes good the deficiency. By applying a theorem of Tarski, it shows that intuitionistic logic is the strongest logic that may be applied, given certain semantic assumptions about vague predicates. The essay ends with an inconclusive discussion of whether those semantic assumptions should be accepted.
\end{abstract}

Keywords Sorites Paradox, Paradox of Sharp Boundaries, intuitionistic logic, topological semantics, open sets, regular open sets, Alfred Tarski, Crispin Wright

Crispin Wright's papers and books have for forty years been read and admired by philosophers throughout the world. He has made, and continues to make, important contributions to debates about vagueness, rulefollowing, realism, scepticism, self-knowledge, the philosophy of mathematics, and the interpretation of Wittgenstein. Just as impressive, to those of us who know him personally, are Wright's qualities as a philosophical interlocutor. Whether in public discussion or private conversation he is quick on the uptake, acute in response, and seemingly incapable of descending to the footling or trivial. One's understanding of a philosophical issue is always deepened by debating it with him.

It is his passion for philosophical debate that led him to found two institutions that have contributed so much to philosophy in Britain and abroad. Philosophers would make the journey to the Arché Centre at St Andrews, and more recently to the Northern Institute of Philosophy at Aberdeen, in 
large part to get the benefit of his reaction to their ideas. The creation of those institutions bears witness to further qualities that are rarely found combined with philosophical distinction: considerable social and administrative energy, and an unquenchable optimism no matter how difficult the times.

It was Wright's essay 'On the Coherence of Vague Predicates' (1975) that first won him a wide readership, and his subsequent papers on vagueness have also made waves. I have no space to survey his work in that area, but one of his recent essays on the topic provides my point of departure. In "'Wang's Paradox",, 1 / his contribution to the Library of Living Philosophers volume that honoured our common teacher, the late Sir Michael Dummett, Crispin Wright presents the most powerful argument I know in favour of the thesis that we should employ intuitionistic logic, not classical logic, when reasoning with vague concepts. $1^{2} /$ For reasons that will emerge, I am not convinced that this thesis is true, but neither am I convinced that it is false. The aim of this paper, which I confess is inconclusive, is to bring into the debate some considerations which ought to inform any eventual decision on the question.

\section{Wright's Paradox of Sharp Boundaries}

The tangled skein that is the Sorites contains several distinct threads. A proper exploration of these and of their relations is impossible here, but one of Wright's contributions has been to focus attention on a form of the paradox that is especially stark and difficult to solve. The discussion in this essay is confined to this version of the problem.

Let us suppose that we have a sequence of a hundred transparent tubes of paint, $a_{1}, \ldots, a_{100}$, with the following properties: tube $a_{1}$ is clearly red; tube $a_{100}$ is clearly orange and hence clearly not red; but for each $n$, tube $a_{n+1}$ is only marginally more orange (and hence only marginally less red) than its predecessor

\footnotetext{
1 The topic of Wright's essay is not Wang's Paradox per se, but rather Dummett's paper 'Wang's Paradox' (Dummett 1975), hence the quotation marks in Wright's title.

2 The idea that intuitionistic logic might help with the paradoxes of vagueness was floated by Hilary Putnam in his 'Vagueness and Alternative Logic' (Putnam 1983). Stephen Read and Crispin Wright (1985) sharply criticized what they took to be Putnam's view, but Putnam (1985) protested that they had misread him. Wright's paper of 2007 may be read as developing the approach to the Sorites that Putnam had floated in 1983 and then presented more clearly in 1985. See also $n .4$ below.
} 
$a_{n}$. Indeed, let us suppose that an observer with good eyesight, when viewing any pair of adjacent tubes $a_{n}$ and $a_{n+1}$ together in white light but without comparing them with other tubes, is unable to perceive any difference in colour between them. That is, we suppose that any two adjacent members of the sequence are indiscriminable in colour. In such a case, the claim that nowhere in the sequence is a red tube immediately followed by a non-red tube seems highly plausible. For if there were a number $N$ for which $a_{N}$ were red while $a_{N+1}$ were not, we would have a pair of tubes which are indiscriminable in colour but where one member is red while the other is not red; this seems to conflict with the correct use of the predicate 'red'. $1^{3} /$ In a semi-formalized language, then, it seems that we may affirm: $\neg \exists n\left(a_{n}\right.$ is red $\wedge \neg\left(a_{n+1}\right.$ is red $\left.)\right)$.

In the situation described, however, we also have

$$
a_{1} \text { is red }
$$

and

$$
\text { (3) } \neg\left(a_{100} \text { is red }\right) \text {. }
$$

Now if we suppose

$$
\text { (4) } \quad a_{99} \text { is red }
$$

then the rule of conjunction-introduction applied to (3) and (4) would yield

\footnotetext{
3 Wright's leading example in his 2007 paper is a Sorites for 'looks red' rather than 'is red'. It is perhaps even clearer that the correct use of 'seems red' precludes the existence of a pair of indiscriminable tubes, one of which seems red while the other does not. However, claim (1) is also highly plausible, and the logical issues I want to address arise equally with 'is red', which is why I have switched to the semantically simpler predicate.
} 


$$
a_{99} \text { is red } \wedge \neg\left(a_{100} \text { is red }\right),
$$

which, by $\exists$-introduction, yields

(6) $\quad \exists n\left(a_{n}\right.$ is red $\wedge \neg\left(a_{n+1}\right.$ is red $\left.)\right)$

which directly contradicts (1). Given (1) and (3), then, supposition (4) stands refuted, so by Reductio we may assert

(7) $\quad \neg\left(a_{99}\right.$ is red).

By repeating this inferential sub-routine a further 98 times, we reach

(8) $\quad \neg\left(a_{1}\right.$ is red $)$

which contradicts (2). This, then, is the initial paradox. We have some reason to accept the trio of postulates (1), (2), and (3), but we also have an apparently valid deduction showing that the trio is inconsistent. It may be noted that the form of Reductio that is applied in reaching line (7) —and that is reapplied at the corresponding later steps - is acceptable to an intuitionist. So the trio comprising (1), (2), and (3) is inconsistent in intuitionistic logic as well as in classical logic.

How should we react to this apparent demonstration of inconsistency? Since the case is one in which (2) and (3) are clearly true, it seems that we must take it as showing that (1) is false. In other words, we would appear to be entitled - indeed, compelled if the question of (1)'s truth arises - to make a further application of the relevant form of Reductio and infer the negation of (1), namely,

(9) $\quad \neg \neg \exists n\left(a_{n}\right.$ is red $\wedge \neg\left(a_{n+1}\right.$ is red $\left.)\right)$. 
In classical logic, however, (9) is equivalent to

$$
\exists n\left(a_{n} \text { is red } \wedge \neg\left(a_{n+1} \text { is red }\right)\right) .
$$

This, however, seems to land us in a yet more acute paradox, one that Crispin Wright has called the Paradox of Sharp Boundaries. For formula (10) says that at some point in the sequence a red tube is immediately followed by a non-red tube, and this seems to ascribe a sharp boundary to the concept red. Some philosophers take the vagueness of a predicate to consist in there not being a sharp boundary between those objects that satisfy it and those objects that do not. But even if the absence of a boundary is not constitutive of vagueness, it still seems absurd to say that one tube is red while a tube indiscriminable in colour from it is not red: little wonder that Wright calls formula (10) the 'Unpalatable Existential'. Yet we have deduced this apparent absurdity by applying the rules of classical logic to the indisputably true premisses (2) and (3).

One initial comment about the argument is in order. Although I have used an existential quantifier in formulating premiss (1) and some subsequent lines of the argument, its employment is entirely dispensable. Since the quantifier ranges over the natural numbers from 1 to 100 , one could replace any statement of the form ' $\exists n \varphi(n)$ ' by the corresponding disjunction ' $\varphi(1) \vee \ldots \vee \varphi(100)$ '. Thus a propositional logic—indeed, a logic of negation, conjunction, and disjunction—suffices to assess the validity of the argument.

The apparent antinomy here has long been a source of scepticism about the applicability of classical logic to deductions involving vague terms, but it is worth distinguishing between two sorts of sceptic. Some hold that the laws of some other logical system codify the standards for assessing the validity of those deductions, while others take a more radical position and deny that such assessments admit of codification at all. Among the radicals was Frege. On his view, statements can stand in law-governed logical relations only if each of their component expressions possesses a reference or Bedeutung. The reference of a predicate is a concept or Begriff. And yet: 
A concept that is not sharply defined is wrongly termed a concept. Such quasi-conceptual constructions cannot be recognized as concepts by logic; it is impossible to lay down precise laws for them. The law of excluded middle is really just another form of the requirement that the concept should have a precise boundary (Frege $1902 \S 56$, p.69).

A few years earlier, he had elaborated the same message in a letter to Peano:

Logic can only recognize sharply delimited concepts. Only under this presupposition can it set up precise laws...Just as it would be impossible for geometry to set up precise laws if it tried to recognize threads as lines and knots in threads as points, so logic must demand sharp boundaries of what it will recognize as a concept unless it wants to renounce all precision and certainty (Frege 1896, 182-3 = Frege 1980, 114-5).

Indeed, Frege diagnoses the Sorites as arising from the application of precise laws to 'quasi-conceptual constructions': 'The fallacy known as the Sorites rests on this: that something (for example, heap) is treated as a concept when logic cannot acknowledge it as such because it is not properly circumscribed' (Frege 1897/8, 165 = Frege 1979, 155).

\section{Intuitionism as the logic of vagueness}

Unlike Frege, Wright does not despair of finding a precise logic for vague reasoning. On his view, though, the logic in question is intuitionistic, not classical (see especially Wright 2007, but also his 2001 and 2003). He reaches this conclusion through a close analysis of the paradoxical argument.

As remarked above, the derivation showing that (1), (2) and (3) form an inconsistent trio is intuitionistically correct; Wright duly accepts that derivation. He further accepts that the statements (2) and (3) are true, so that the derivation shows that (1) is false. But he insists that that conclusion is acceptable. We are tempted to assert (1) because we think we know that there is no sharp cut-off between the red 
members of the sequence and the non-red members. But really, we do not know any such thing. In order to assert (1) - i.e., in order to assert that there is no sharp cut-off — we would require an insight into the semantics of the predicate 'red'-i.e., an understanding of the way it contributes to the determination of the truth or falsity of statements containing it - that we do not (yet) possess. Of course, we are equally unable to assert that there is any sharp cut-off point between the red and the non-red tubes in our sequence, but lack of entitlement to assert should not be confused with entitlement to deny. On a correct view, Wright claims, a predicate's 'vagueness...does not consist in the absence of sharp cut-offs'; rather, it consists in there being 'nothing in our practice with the predicate that grounds the claim that there is a sharp cut-off' (Wright 2007, 439). In particular, where we have a simple statement in which a vague predicate is applied to one of its borderline cases,

the breakdown in convergence of verdicts leaves us without uncontroverted evidence for its truth or for the truth of its negation. Since we lack convincing theoretical grounds to think that one or the other must be true nonetheless - because such grounds would have to regard the determinants of truth and falsity as constituted elsewhere than in our linguistic practice-we are left with no compelling reason to regard either [the statement] or its negation as true (Wright op. cit., 441).

Let us pick a tube from the middle of our sequence- $-a_{50}$, say. That tube, we may suppose, is a borderline case of redness: even given full information about its colour, and full information about how competent English speakers are disposed to use the predicate 'red', it remains indeterminate whether the predicate correctly applies to the tube. On Wright's view, we should refrain both from asserting and from denying 'Tube $a_{50}$ is red'. Similarly, he thinks, we should refrain both from asserting and from denying 'Some red tube in the sequence is immediately followed by a non-red tube'-that is, we should refrain both from asserting and from denying formula (10). In particular, then, we should refrain from denying (10); equivalently, we should refrain from asserting (1).

What, though, of the second part of the paradoxical argument? As remarked, (1), (2) and (3) are (intuitionistically) inconsistent, and premisses (2) and (3) are clearly true. So it seems that (1) is false. But 
if (1) is false, then its negation is true. So we are entitled to assert-indeed, we are committed to asserting-(9):

$$
\neg \neg \exists n\left(a_{n} \text { is red } \wedge \neg\left(a_{n+1} \text { is red }\right)\right) .
$$

Wright accepts (9). Because (1) — the denial that red has a sharp cut-off point—contradicts the evident truths (2) and (3), we must be prepared to deny any denial that there is a sharp cut-off point. Assuming that denying a statement is equivalent to affirming its negation, it follows that we must be prepared to affirm the doubly negated formula (9). However, in order to reach the genuinely unpalatable (10), which asserts that there is a sharp cut-off between the red and the non-red tubes, we would need to eliminate a double negation. And this, Wright argues, we are neither compelled nor entitled to do. Since we cannot assume that statements involving vague expressions are either true or false, 'we should...abstain from unrestricted use of the law of double negation elimination' (ibid.). Such abstention enables us consistently to deny (1), assert (9), but refrain from asserting or denying (10). This combination of assertions, denials, and abstentions from assertion and denial constitutes, Wright proposes, a coherent response both to the Sorites proper and to the related Paradox of Sharp Boundaries. $1^{4} /$

We have here a novel and interesting argument for intuitionistic logic. Pace Frege, there is a logic for assessing deductions involving vague predicates, but the logic in question is intuitionistic rather than classical. If it works at all, Wright's argument has very wide application. Many ordinary predicates are such that nothing in our ordinary practice with them grounds the claim that their extensions have sharp cutoffs. For many of those predicates, indeed, it will be possible to construct a Sorites sequence. To those predicates, Wright's argument will apply: on pain of contradicting clear truths, we must deny that there is no sharp cut-off point, whilst resisting the unsupported assertion that there is such a cut-off. This position

\footnotetext{
${ }^{4}$ The kernel of Wright's position, then, is to deny the denial of (10) while refraining from either asserting or denying (10) itself. This is why I read Wright's essay of 2007 as elaborating the position Putnam took in 1985: 'In my proposal (10) is not denied. What the Read-Wright argument does show, in fact, is that I should deny (10)'s denial - that is, I should accept the double negation of (10)' (Putnam 1985, 203, with re-numbering of formulae). The double negation of (10) is (9) which, as we have seen, Wright does accept in his 2007 paper.
} 
is stable only if there are restrictions on the elimination of double negations; so we have an argument in favour of a logic (like intuitionistic logic) which imposes such restrictions.

What should we make of this argument? In his reply to “"Wang's Paradox", Michael Dummett put on record his 'admiration for the beautiful solution of the Sorites advocated by Crispin Wright, clouded by a persistent doubt whether it is correct' (Dummett 2007a, 453). Anyone who appreciates logicophilosophical finesse will share Dummett's admiration for an ingenious proposal, but there are, indeed, two immediate reasons to doubt its correctness.

First, Wright moves too quickly from the observation that we hesitate when pressed to say whether a borderline red-orange object is red, to what is really needed to sustain the distinction the intuitionist wishes to draw between (9) and (10). The observation is correct, and if one further supposes that the only possible grounds for the truth of a vague statement must be overt in our ordinary practices of asserting and denying it, then it cannot be assumed that an atomic statement in which a vague predicate is applied to one of its borderline cases is either true or false. This point, however, is not immediately relevant to the status of the formulae (9) and (10). These formulae are not atoms, and (on intuitionistic principles) we may assert that a complex statement is bivalent even when we cannot assert the bivalence of any of its components. $I^{5} /$ So we need a further argument to show that we cannot assert the bivalence of (9) and (10).

Wright seems to have in mind a traditional Heyting-style semantics for the language of intuitionism on which truth goes with assertibility and bivalence goes with decidability. $1^{6} \%$ Under that semantics, the existentially quantified formula (10) may be assumed to be bivalent if (a) the relevant domain of quantification is surveyable, and (b) each matrix instance is decidable. Condition (a) is surely met. In a Sorites deduction, the relevant sequence of objects is finite; indeed, as noted, the deduction would go through in essentially the same way if (10) were replaced by a disjunction. So Wright must deny that all the matrix instances of (10) are decidable; in particular, he must deny that we have a general ground

\footnotetext{
${ }^{5}$ For example, an intuitionist is always entitled to assert the falsity, and hence the bivalence, of $\neg(A \vee \neg A)$, whatever the status of $A$.

${ }^{6}$ See especially the discussion on p.441 of Wright 2007, which concludes: 'We should therefore abstain from unrestricted use of the law of double negation elimination. We do so on the grounds that it is nonconservative of knowledge- exactly the Intuitionistic reservation'.
} 
for denying any formula of the form ' $a_{n}$ is red $\wedge \neg\left(a_{n+1}\right.$ is red $)$ '. For, in a Heyting-style semantics, such a ground would render (10) false, so we would have a proof of (1), as well as of (9), and Wright's way out of paradox would collapse back into contradiction. The problem is that there is a plausible general ground for denying any such conjunction—namely, Timothy Williamson's thesis that knowledge of inexact matters demands margins for error (Williamson 1994, 226-30). According to that thesis, knowledge that $a_{n}$ is red requires that $a_{n+1}$ may also be red, for all we know; this excludes knowledge that $a_{n+1}$ is not red. Since Wright takes it that an entitlement to assert requires knowledge, Williamson's thesis provides a general ground for rejecting any assertion of the form ' $a_{n}$ is red $\wedge \neg\left(a_{n+1}\right.$ is red $)$ '. On Heyting's principles, this would amount to a ground for denying (i.e., affirming the negation of) any such conjunction. This is turn yields a ground for deeming (10) to be false, which is inconsistent with Wright's claim that its negation, (1), is also false. If he wishes to persist with a Heyting-style semantics, Wright badly needs to dispel the considerable attractions of the thesis that inexact knowledge demands margins for error, for that thesis brings his position into ruin. $1^{7} /$

Second, and more importantly, Wright's suggestion that the logic of vague statements is intuitionistic is not sustained by any detailed semantic analysis. He points to broad analogies between the intuitionists' attitude to mathematical statements and the attitude that he recommends taking towards vague statements: in each case, statements' 'truth and falsity have to be thought of as determined by our very practice, rather than by principles which notionally underlie' that practice. $1^{8} /$ As Dummett has remarked, however, it is most implausible to suppose that the Heyting semantics for the language of intuitionist mathematics correctly describes the contribution that the connectives and quantifiers make to the meaning of vague statements:

\footnotetext{
7 This thesis about knowledge does not entail Williamson's own epistemicism about vagueness-the claim that there is a sharp cut-off point in the sequence of red things, but that we cannot in principle know where it is. Someone who denies, or refrains from accepting, that there is a sharp cut-off can still hold that knowledge that such-and-such an object is red requires a margin for error.

8 Wright 2007, 441. One of Wright's theses in his essay is that competence with a word like 'red' is not underpinned or informed by adherence to any rule. If that thesis is correct, then a semantic theorist cannot sensibly aim to articulate the rules that govern competence with 'red'. But in calling for a more detailed semantic analysis than Wright provides, I am not asking for that, but only for something that it is reasonable to seek on any plausible view. For the predicates 'is not red', 'is either red or orange', etc. are clearly semantically complex, so it must be in order to ask for a statement of the general principles that relate their meanings to the meanings of their components. That is what I mean by a semantic analysis.
} 
Wright can hardly say that to grasp the meaning of the statement ['This curtain is red', said of a borderline red-orange curtain] is to be able to recognize a demonstration that the colour of the curtain is red, not orange, or that it is orange, not red. Presumably he must say that it consists in an ability to judge that the colour of the curtain is neither definitely red nor definitely orange, but is definitely either one or the other. If so, however, the theory of meaning for vague statements on which Wright wishes to rest intuitionist logic as applying to them interprets disjunction quite differently from the intuitionist theory of meaning for mathematical statements (Dummett 2007, 451-2)

As we shall soon have occasion to note, Heyting's is far from being the only semantic theory that sustains intuitionistic logic, but Dummett's general point remains: 'It is not enough to show that the Sorites paradox can be evaded by the use of intuitionistic logic; what is needed is a theory of meaning, or at least a semantics, for sentences containing vague expressions that shows why intuitionistic logic is appropriate for them rather than any other logic' (op. cit., 453).

Wright might, I suppose, contest the need for a semantic theory. At least, he might argue that the construction of such a theory is not needed to vindicate his use of intuitionistic logic. We are dealing, after all, with a paradox: statements involving vague terms which we are disposed to assert yield contradictions given classical logic, but they do not yield absurdities when the operative logic is intuitionistic. One might reasonably think that this fact provides strong reason for restricting some classical laws even in advance of constructing a semantic theory. Indeed, Wright might pray in aid some of Dummett's own writings here. At the end of the chapter in The Logical Basis of Metaphysics which presents his reasons for adopting intuitionistic logic, Dummett remarks that 'the meanings of the intuitionistic logical constants can be explained in a very direct way, without any apparatus of semantic theory, in terms of the use made of them in practice' (Dummett 1991, 299). 'A metalanguage whose underlying logic is intuitionistic', he goes on, 'can be understood, and its logical laws acknowledged, without appeal to any semantic theory and with only a very general meaning-theoretical background. If that is not the right logic, at least it may serve as a medium by means of which to discuss other logics' (op. cit., 300). The 'very general meaning-theoretical 
background' to which Dummett refers includes the requirement of 'harmony' between the introduction and elimination rules for a connective. Wright might fairly point out that the requirement of avoiding outright contradiction is more clearly compelling than that of harmony, and that the former requirement is all he really needs to press his case for intuitionistic logic as the 'right logic' when reasoning with vague terms.

For all that, a semantic theory remains desirable: it may help us to understand why the distinctively classical rules are invalid when applied to vague terms. As Dummett was wont to say in the rather different context of the foundations of mathematics, to observe that a certain postulate leads to contradiction is to 'wield the big stick' when what is really wanted is some explanation of why the postulate is troublesome.

In this paper, I want to take up Dummett's challenge and construct a semantic theory for vague terms which offers some hope of vindicating intuitionism as the logic of vagueness. I am not sure that the semantic theory I propose is fully satisfactory; indeed, $\$ 5$ will present some reasons for doubting it. Those reasons, however, are far from conclusive, and it would be interesting to know whether Crispin Wright is disposed to accept it. If he were, it would give him a way of meeting Dummett's challenge head on by showing why intuitionistic logic, rather than any other logic, sets the standards for assessing deductive arguments involving vague terms.

\section{A semantics for vague predicates that validates intuitionistic logic}

The semantic theory I have in mind is really nothing more than an application of a theorem of Alfred Tarski's.

Topologists have the notion of an open set. The mark of such a set is that, whenever an object belongs to it, there is a 'neighbourhood' around the object, all of whose members also belong to the set. Suppose we have a domain which comprises all the possible coloured objects, not merely those that actually exist. Then it is very natural to postulate that the set of red objects in this domain will be open in a suitably defined topology. For suppose that a possible object $x$ is red. Then it is natural to postulate that there is a neighbourhood of possible objects around $x$ that are also red. An object's perceived colour does not depend only on the wavelength of the light it reflects or emits: it also depends on the ambient 
illumination and on the colours of nearby objects. All the same, if our red object, $x$, reflects light of wavelength $700 \mathrm{~nm}$, then it is plausible to claim that all the possible objects which are observed in the same context as $x$ and in circumstances where human colour perception is as it actually is, but which reflect light of wavelengths between 699 and $701 \mathrm{~nm}$, will also be red. In a suitably defined topology, then, these possible objects will constitute a neighbourhood around $x$, all of whose members are also red. An object that reflects light of $700 \mathrm{~nm}$ is a paradigm case of redness, but the argument may be adapted to show that there is a neighbourhood around any red object. Suppose that $y$ is a red object that reflects light of $620 \mathrm{~nm}$, and so is close in colour to some orange objects. Even assuming that colour perception is as it actually is, it may well be that some possible objects that reflect light of wavelengths between 619 and $621 \mathrm{~nm}$ are not red. However, so long as $y$ is red, it is plausible to claim that there is some neighbourhood around $y$ - for example, those possible objects that are viewed in the same context as $y$ but which reflect light of wavelengths between 619.9999 and $620.0001 \mathrm{~nm}$ - all of whose members are red. This smaller neighbourhood is enough to ensure that the set of red objects in the domain is open. It is also natural to postulate that the set of possible objects that are not red will be another open set in the same topology. For if $z$ is a possible object that is not red, perhaps because it reflects light of only $550 \mathrm{~nm}$, it is plausible to claim that all the possible objects which are viewed in the same context as $z$, but which reflect light of wavelengths between 549 and $551 \mathrm{~nm}$, are also not red.

Arguments analogous to that just sketched may be advanced for many vague predicates. Suppose that $x$ is a tall man in the domain of all possible men. Then, whatever precise height $x$ has, it is plausible to claim that there is a neighbourhood around $x$ all of whose members are also tall men. If $x$ is close to the borderline for being tall, the members of the neighbourhood may have a precise height that is very close to $x$ 's. All the same, the set of tall possible men will be open in an appropriately defined topology. So will the set of possible men who are not tall.

We cannot assume that the set of actual men who are tall is open in the relevant topology. Suppose that every man of height greater than $5^{\prime} 8^{\prime \prime}$ dies, except for one man, Adam, whose height is $6^{\prime} 1^{\prime \prime}$. Adam is tall, but in the circumstance envisaged there is no neighbourhood of actual tall men around him. All the same, it is entirely natural to take account of merely possible existents when giving a semantic theory for predicates. This is certainly the case when we are concerned with logical relations between 
statements and predicates. When a conclusive statement follows logically from some premiss statements, it is logically necessary that it is true whenever they are all true. Similarly, when a conclusive predicate follows logically from some premiss predicates, it is logically necessary that it is true of whatever they are all true of. Given that, it is natural to take the semantic value of a predicate to be a subset of a domain of all logically possible objects, not merely the actual ones. This raises, of course, the vexed question of how we might best conceive of that domain, but there is a way of bracketing that question for present purposes. Rather than taking the semantic value of 'tall' to be a subset of the domain of all possible men, we might take it to be a set of specific heights, determined by a real number (with 'foot' as the unit, say): a man will be tall if his specific height belongs to this set. Since a specific height will exist even if no man has that height, this semantic value of 'tall' will be an open set regardless of men's heights. This manoeuvre also enables to be specific about the topology in question: on this approach, the semantic value of 'tall' is an open set in the standard topology on the real line. Similarly, we may take the semantic value of 'red' to be an open set in the same topology, where the real numbers in this case specify wavelengths of reflected or emitted light. An object may be said to be 'red' if it reflects or emits light of a wavelength belonging to this semantic value. These assignments of semantic values are of course idealized. No determinate set of real numbers, whatever its topological properties, contains the heights (in feet) of all and only tall men; and an object's perceived colour depends on the ambient illumination and the colours of nearby objects as well as on the wavelengths of light it emits or reflects. But all the semantic theories we currently have involve considerable idealization and (as we shall see) the present idealization leads to very different results from the familiar one, which postulates sharp boundaries even for vague predicates such as 'red' and 'tall'.

How might these ruminations about open sets encourage a theorist who recommends using intuitionistic logic when assessing deductive arguments involving vague terms? Where $S$ is any topological space, and $L$ is any propositional language whose connectives are ' $\wedge$ ', ' $\vee$ ', ' $\rightarrow$ ', and ' $\neg$ ', let us say that a map $v$ is an interpretation of $L$ into $S$ if $v$ maps each well-formed formula of $L$ into an open subset of $S$ in such a way that

$$
\begin{aligned}
& v(A \wedge B)=v(A) \cap v(B) \\
& v(A \vee B)=v(A) \cup v(B)
\end{aligned}
$$




$$
v(A \rightarrow B)=\operatorname{Int}(v(B) \cup(S-v(A)))
$$

and $\quad v(\neg A)=\operatorname{Int}(S-v(A))$.

(Thus $v(\neg A)=v(A \rightarrow \perp)$, where $v(\perp)=\varnothing$.) The interior Int $(X)$ of a set $X$ is the largest open set contained in $X$. Accordingly, the clauses for the conditional and for negation ensure that a conditional or negated formula is mapped into an open set in $S$. Since the intersection and union of two open sets are also open, $v$ maps every statement in $L$ to an open set in $S$. Let us then say that an $\operatorname{argument}$ ' $A_{1}, \ldots, A_{n}$; so $B$ ' in $L$ is valid in the topological space $S$ if and only if

for every interpretation $v$ of $L$ into $S, v\left(A_{1}\right) \cap \ldots \cap v\left(A_{n}\right) \cap S \subseteq v(B)$.

Let us define a consequence relation $A_{1}, \ldots, A_{n} \aleph_{T o p} B$ as one which obtains when the argument ' $A_{1}, \ldots, A_{n}$; so $B^{\prime}$ is valid in every topological space. A theorem due to Tarski-the Second Principal Theorem of his paper 'Sentential Calculus and Topology' $(1938,448)$ — then tells us that the intuitionistic propositional calculus is sound and complete with respect to this notion of consequence. That is, where IInt $_{\text {signifies }}$ deducibility by the rules of the intuitionistic propositional calculus,

$$
A_{1}, \ldots,\left.A_{n}\right|_{\text {Int }} B \text { if and only if } A_{1}, \ldots,\left.A_{n}\right|_{T o p} B
$$

In fact, this Theorem of Tarski's tells us more. Let us define the $\operatorname{closure~} C l(X)$ of a set $X$ in a topological space $S$ to be the complement of the interior of the complement of $X$. That is, $C l(X)=$ $S-(\operatorname{Int}(S-X))$. Let us call a topological space $S$ dense-in-itself when, for every $x \in S, x \in C l(S-\{x\})$. Let us call $S$ normal when, for any two subsets $X$ and $Y$ of $S$, if $C l(X)$ is disjoint from $C l(Y)$ there exist disjoint open subsets $U$ and $V$ of $S$ such that $C l(X) \subseteq U$ and $C l(Y) \subseteq V$. Let us say that $S$ has a countable basis if there is a countable family $U=\left\{U_{i}\right\}_{i \in \omega}$ of open subsets of $S$ such that every non-empty open subset of $S$ can be represented as the union of a sub-family of $U$. Finally, let us say that a topological space is Euclidean if it is dense-in-itself, normal, and has a countable basis. Then Tarski (ibid.) also proved 
(B) Where $S$ is any given Euclidean topological space, $A_{1}, \ldots, A_{n} \vdash_{I n t} B$ if and only if the argument ' $A_{1}, \ldots, A_{n}$; so $B$ ' is valid in $S$.

The $n$-dimensional Euclidean space $\mathfrak{R}^{n}$ with its usual topology is Euclidean in the present sense. Although the usual basis of open balls in $\mathfrak{R}^{n}$ is not countable, every open set in $\mathfrak{R}^{n}$ can be represented as the union of a sub-family of the restricted basis comprising those open balls with rational radii and whose centres have rational Cartesian coordinates; this restricted basis is countable. $I^{9} /$ In particular, then, $(B)$ tells us that $A_{1}, \ldots, A_{n} F_{\text {Int }} B$ if and only if the argument ' $A_{1}, \ldots, A_{n}$; so $B$ ' is valid in the usual topology on the real line $\mathfrak{R}$. This result immediately shows that ${ }^{\lceil} A \vee \neg A^{\rceil}$is not a theorem of the intuitionistic sentential calculus and that $\neg \neg A \vdash A$ is not a correct sequent in that calculus. For consider a sentence letter $P$ whose value $v(P)$ in $\mathfrak{R}$ is the open half-line $(0, \infty)$. Then $v(\neg P)=\operatorname{Int}(\mathfrak{R}-v(P))=\operatorname{Int}((-\infty, 0])=(-\infty, 0)$. Thus $v(P \vee \neg P)=v(P) \cup v(\neg P)=(0, \infty) \cup(-\infty, 0)$, so that ${ }^{\lceil} P \vee \neg P^{\rceil}$does not meet the necessary condition for validity, viz. $\mathfrak{R} \subseteq v(P \vee \neg P)$. To show the invalidity of $\neg \neg A \vdash A$, consider a sentence letter $Q$ whose value $v(Q)$ is set to be $(-\infty, 0) \cup(0, \infty)$. In that case, $v(\neg Q)=\operatorname{Int}(\Re-v(Q))=\operatorname{Int}(\Re-((-\infty, 0) \cup(0, \infty)))=$ Int $(\{0\})=\varnothing$. So $v(\neg \neg Q)=\operatorname{Int}(\Re-v(\neg Q))=\operatorname{Int}(\mathfrak{R}-\varnothing)=\operatorname{Int}(\Re)=\Re$. So the sequent $\neg \neg Q \vdash Q$ does not meet the necessary condition for validity, viz. $v(\neg \neg Q) \subseteq v(Q)$.

In fact, Tarski's proof of this Theorem may be adapted to establish corresponding results about monadic predicates rather than closed formulae. Let $L^{\prime}$ be a language consisting of a number of such predicates, formed by freely applying the predicate-forming operators 'and', 'or', 'if...then' , and 'not' to a stock of atomic monadic predicates. $L^{\prime}$, then, contains no singular terms or quantifiers. Indeed, it contains no closed formulae: ${ }^{\lceil} A$ and $\left.B\right\rceil$ is a conjunction of two monadic predicates, meaning (as it might be) 'red and spherical'. That need not stop us from applying deductive rules to the predicates in $L^{\prime}$ : it makes sense to say, for example, that 'red and spherical' entails 'red'. Let us consider in particular the deductive system in which the rules of the intuitionistic propositional calculus are applied to these simple and complex monadic predicates, and let $F_{I n t}$ 'signify deducibility by the rules of this system. Where $S$ is again any topological

9 So, for example, in the usual topology on $\mathfrak{R}$, the open set $(0, \sqrt{ } 2)$ is the union of the countable collection of open sets $(0, r)$, where $r$ is a positive rational number such $r^{2}<2$. 
space, we say that a map $v$ is an interpretation of $L^{\prime}$ into $S$ if $v$ maps each predicate in $L^{\prime}$ into an open subset of $S$ in such a way that $v(A$ and $B)=v(A) \cap v(B), v(A$ or $B)=v(A) \cup v(B), v($ if $A$ then $B)=$ Int $(v(B) \cup(S-v(A)))$, and $v(\operatorname{not} A)=\operatorname{Int}(S-v(A))$. As before, we say that an argument ' $A_{1}, \ldots, A_{n}$; so $B$ ' in $L^{\prime}$ is valid in the topological space $S$ if and only if

for every interpretation $v$ of $L^{\prime}$ into $S, v\left(A_{1}\right) \cap \ldots \cap v\left(A_{n}\right) \cap S \subseteq v(B)$,

and we define a consequence relation $A_{1}, \ldots, A_{n} k_{T o p^{\prime}} B$ as one which obtains when the $L$ ' argument ' $A_{1}, \ldots$, $A_{n}$; so $B$ ' is valid in every topological space. Then Tarski's Second Principal Theorem also yields

$$
A_{1}, \ldots, A_{n} \vdash_{I n t^{\prime}} B \text { if and only if } A_{1}, \ldots, A_{n} \vDash_{T o p^{\prime}} B
$$

and

$\left(B^{\prime}\right) \quad$ Where $S$ is any given Euclidean topological space, $A_{1}, \ldots, A_{n} \vdash_{\text {Int' }} B$ if and only if the $L$ ' argument ' $A_{1}, \ldots, A_{n}$; so $B$ ' is valid in $S$.

Which of these four deliverances of Tarski's Theorem may help to vindicate the suggestion that intuitionistic logic is the logic of vagueness? The ones to look at are $\left(A^{\prime}\right)$ and $\left(B^{\prime}\right)$. The intuitive idea we are exploring is that the extension of a vague predicate may be expected to be an open set in a suitably defined topology. It is the topological interpretation of $L^{\prime}$ that directly bears on that idea, not the interpretation of $L$. We shall eventually have to return to the logic of vague statements. Even if we could show that an intuitionistic system was the right logic for assessing deductions involving vague monadic predicates, this would not vindicate Wright's solution to the Sorites, which requires a propositional logic, not merely a logic for monadic predicates. All the same, the intuitive idea invites us to reverse the usual order of explanation and start by considering the logical behaviour of predicates rather than the behaviour of complete statements. 
What, then, might $\left(A^{\prime}\right)$ tell us about the logic of vague predicates? It tells us that when a monadic predicate $B$ is not intuitionistically deducible from some premiss predicates $A_{1}, \ldots, A_{n}$, there is some topological space $S$, and some interpretation $v$ from $L^{\prime}$ into $S$, for which $v\left(A_{1}\right) \cap \ldots \cap v\left(A_{n}\right) \cap S$ is not a subset of $v(B)$. As things stand, though, we do not know that this particular topological space $S$ is one in which a vague predicate has an open extension. Theorem $\left(A^{\prime}\right)$, then, is unlikely to be effective in showing that intuitionistic logic is the strongest logic that may legitimately be employed in deductions involving vague predicates. I shall therefore explore the possibility suggested by $\left(B^{\prime}\right)$ - that the standards for assessing such a deduction are set by some particular Euclidean space, determined by the deduction's context. ${ }^{10} \%$

Given our discussion so far, certain Euclidean spaces suggest themselves as setting the standard for assessing deductions involving vague predicates. Let us suppose that the atomic predicates of $L^{\prime}$ are the colour terms 'red' and 'orange'. As we have seen, if we take the semantic values of these terms to be sets of precise wavelengths of light, then (a) the semantic value of each predicate in $L^{\prime}$ (whether simple or complex) will be an open set in the natural topology on $\Re$ and (b) that topology is Euclidean. Accordingly, $\left(B^{\prime}\right)$ tells us that the logic of $L^{\prime}$ will be intuitionistic so long as the following holds:

An argument ' $A_{1}, \ldots, A_{n}$; so $B$ ' in $L$ ' is valid simpliciter if and only if it is valid in $\Re$.

Given the idealizations already made, this account of validity is prima facie plausible. We are supposing that the semantic values of colour terms are open sets in $\mathfrak{R}$. Under that supposition, the conditions on $v$ would appear to be faithful to the meanings of 'and', 'or', 'if...then' and 'not' as these expressions connect predicates. These considerations by no means establish the proposed account of validity, but they do make it worth exploring in detail.

Similar considerations apply when other vague terms stand as the atomic predicates in $L^{\prime}$. Suppose that the atomic predicates are now 'short' and 'tall' (as applied to men). As before, we take the

${ }^{10}$ In discussing Wright's hypothesis in my book The Boundary Stones of Thought (Rumfitt 2015, §8.3), I explored only the possibility of vindicating it by way of $\left(A^{\prime}\right)$. Accordingly, the rejection of the hypothesis in that book was premature. 
semantic values of these terms to be sets of real numbers, which measure men's precise heights (in feet, say); a man will be tall if his precise height belongs to the value of 'tall'. In this case, too, (a) the semantic value of each predicate in $L^{\prime}$ (whether simple or complex) will be an open set in the natural topology on $\mathfrak{R}$ and (b) that topology is Euclidean. Given these idealizing assumptions, it is again plausible to hold:

An argument in $L^{\prime}$ is valid simpliciter if and only if it is valid in $\mathfrak{R}$.

It then follows from $\left(B^{\prime}\right)$ that the logic of $L^{\prime}$ will be intuitionistic.

All this might encourage the following Conjecture:

An argument moving from vague predicate premisses to a vague predicate conclusion is valid simpliciter if and only if it is valid in some particular Euclidean space.

If the Conjecture were correct, then intuitionistic logic would indeed be the right logic to use in assessing the validity of such arguments.

\section{The Sorites Revisited}

Even if the semantic values of vague predicates are always open sets in a Euclidean topology, the Conjecture may still be false. The Conjecture also requires that having its semantic value be an open set in a Euclidean topology is the strongest topological condition that may be imposed on a vague predicate. If the semantic values of such predicates have to satisfy a stronger topological condition, that condition might well sustain a stronger-than-intuitionistic logic. In the next section, I shall consider whether the Conjecture is vulnerable to objection along these lines. On any view, however, our analysis is incomplete. If the Conjecture is correct, then intuitionistic logic will set the standards for assessing deductions in which we move from some vague (monadic) predicates as premisses to a conclusion which also a monadic predicate. Intuitionistic logic, in other words, regulates such deductions as 'Red and large; so red'. It remains an open 
question, however, whether that logic also regulates the paradoxical deduction of $\S 1$. In that argument, ' $\wedge$ ', ' $\vee$ ', and ' $\neg$ ' are all used to connect complete sentences, not predicates, and it is essential that they should be so used. A step in a Sorites argument cannot be analysed as an inference from premisses saying that a given object has such-and-such a property, to the conclusion that the same object has some further property: the thrust of the Sorites is to argue for assigning a given vague property to a newly considered object. So the logic to be employed in evaluating the Sorites is moot until we have extended our account of the meanings of 'and', 'or', 'if...then', and 'not' to yield an account of the meanings of ' $\wedge$ ', ' $\vee$ ', ' $\rightarrow$ ', and ' $\neg$ '. We do not, however, need to extend our theory further and explain the meanings of quantified formulae. Instead we can replace existentially quantified formulae such as Wright's (10) with disjunctions such as $\left(10^{\prime}\right)$. In what follows, I shall write as though the deduction of $\S 1$ has been recast in just this way, so that it is the rule of $\vee$-introduction, not $\exists$-introduction, which validates the step from $\left(5^{\prime}\right)$ to $\left(6^{\prime}\right)$.

How, then, might we extend our semantics for 'and' etc. so as to yield a semantics for ' $\wedge$ ' etc.? There is a natural way of doing this. Consider the formula ' $a_{1}$ is red $\wedge \neg\left(a_{2}\right.$ is red $)$ ' as an example. That sentence is true if and only if the two-place predicate ' $\xi_{1}$ is red and not ( $\xi_{2}$ is red)' is true of the ordered pair $\left\langle a_{1}, a_{2}>\right.$. Given that the semantic value $V_{1}$ of ' $\xi_{1}$ is red' is an open set in $\mathfrak{R}$ with its usual topology, the Cartesian product $V_{1} \times \mathfrak{R}$ will be an open set in $\mathfrak{R}^{2}$ with its usual topology. Similarly, given that the semantic value $V_{2}$ of 'not ( $\xi_{2}$ is red)' is an open set in $\mathfrak{R}$ with its usual topology, the Cartesian product $\mathfrak{R} \times V_{2}$ will be an open set in $\mathfrak{R}^{2}$ with its usual topology. So $V_{1} \cap V_{2}=\left(V_{1} \times \mathfrak{R}\right) \cap\left(\Re \times V_{2}\right)$ will be another open set in $\mathfrak{R}^{2}$. As noted, ' $a_{1}$ is red $\wedge \neg\left(a_{2}\right.$ is red $)$ ' is true if and only if $\left\langle a_{1}, a_{2}\right\rangle \in V_{1} \cap V_{2}$. Now in addition to the sentential connectives ' $\wedge$ ', ' $\vee$ ', ' $\rightarrow$ ' and ' $\neg$ ', ${ }^{11} /$ the language $L$ of our Sorites argument consists of a single atomic predicate, 'red', together with a hundred singular terms: 'tube $a_{1}$ ', .., 'tube $a_{100}$ '. By extending the method just indicated, we can construct, for any sentence $A$ in $L$, a one-hundred place predicate $P$ such that $A$ is true if and only if $P$ is true of $\left\langle a_{1}, \ldots, a_{100}\right\rangle$, where the semantic value of $P$ is always an open set in $\mathfrak{R}^{100}$ with its usual topology. The predicate $P$ will belong to a language whose atomic

11 The particular Sorites deduction given in $\S 1$ happens not to contain ' $\rightarrow$ ' but other such deductions will contain the conditional, so it needs to be treated: in intuitionistic logic, ' $\rightarrow$ ' cannot be defined in terms of the other connectives. 
predicates ascribe redness to various members of sequences of one hundred objects (thus one atom will say 'First member is red, second member is red, third member is red'), and whose only compositional devices are the predicate connectives 'and', 'or', 'if...then', and 'not', interpreted to signify intersection, union, interior of relative complement, and interior of complement. The construction is such that a deduction ' $A_{1}, \ldots, A_{n}$; so $B$ ' in $L$ is valid if and only if the corresponding deduction involving one-hundred place predicates, ' $B_{1}, \ldots, B_{n}$; so $Q$ ', is valid in $\mathfrak{R}^{100} . \mathfrak{R}^{100}$ with its usual topology is another Euclidean space. So, by $\left(B^{\prime}\right)$, we conclude that intuitionistic logic sets the standard for assessing deductions in $L$ as well as for deductions in $L^{\prime}$.

The method used to construct the predicates is of course inspired by Tarski's insight that a closed formula in a language with quantifiers is true if and only if it is satisfied by every infinite sequence of objects in the domain (see Tarski 1935, 195, Definition 23). Because, however, we are dealing with a language with sentential connectives, not quantifiers, we need consider only finite sequences: any deduction is finite and so will contain only finitely many singular terms.

The analysis above could be applied to many Sorites deductions. A Sorites argument for 'tall' may be analysed in essentially the same way, given that the semantic value for the monadic predicate 'tall' is another open set in $\mathfrak{R}$. Of course, the analysis rests on the Conjecture. Given that, however, it provides a strong case for Wright's hypothesis that intuitionistic logic regulates deductions involving vague predicates. Given the master assumption that the semantic values of vague predicates are open sets, the semantic principles for 'and', 'or', 'if...then', and 'not' are natural—indeed, they are well-nigh inevitable. Moreover, since $\mathfrak{R}$ and all its finite powers provide Euclidean topologies in which many vague predicates have open sets as their semantic values, the analysis extends to account for the validity of deductions involving different sorts of vague predicate: the analysis accounts for the validity of 'John is either rich or tall; John is not tall; so John is rich' as easily as it accounts for the validity of 'Tube $a$ is either red or orange; tube $a$ is not orange; so tube $a$ is red'. In defending his hypothesis, Wright can say more than that adopting intuitionistic logic staves off contradiction in the Paradox of Sharp Boundaries. The semantic theory just sketched provides what Dummett was missing: an explanation of why intuitionistic logic is the right logic to use in assessing deductions involving vague predicates. 


\section{Problems for the Proposal}

Despite these attractive features, the suggested explanation faces problems. The problems may not be insuperable, but I shall conclude by mentioning the two which seem to me to be the most serious.

The first is that Tarski proved the Theorem on which the semantic analysis rests in a classical metalogic: at various points in his proof, he employs classical rules of inference that are not acceptable to an intuitionist. In one respect, this casts further doubt on the thesis that classical logic provides the standards for assessing deductions involving vague predicates: even if we assume classical principles in the metalogic, the semantic theses we have been considering validate a weaker-than-classical logic for the object language. In the end, though, we shall want to prove that intuitionistic logic is sound and complete with respect to the proposed semantics in an intuitionistic metalogic. The thesis, after all, is that intuitionistic logic is the logic of vagueness, and the metalanguage in which the semantic theory is cast is just as vague as the object language. The colour predicate 'red' is vague, but so is the metalinguistic predicate 'satisfies the predicate "red"'. I do not know whether Tarski's Theorem—or, more particularly, its consequence $\left(B^{\prime}\right)$ - can be proven in an intuitionistic metalogic, but this question is important for the vindication of Wright's hypothesis. The claim that intuitionistic logic is the logic of vagueness would be better supported if the answer were affirmative.

The other major problem the theory faces concerns the Conjecture itself. As I argued at the start of $\S 3$, it is highly plausible to claim that the semantic value of a vague predicate will be an open set in a suitably defined topology. By itself, though, this claim does not sustain the Conjecture. We also need it to be the case that the strongest topological condition which may be imposed on a vague predicate is that its semantic value should be an open set in a Euclidean topology. There are reasons to doubt that this holds in general. Indeed, there are reasons to doubt that it holds even in the particular cases we have been examining. For how did we show that Excluded Middle and Double Negation Elimination were not valid in $\Re$ ? In each case, the proofs involved assigning the subset $(0, \infty) \cup(-\infty, 0)$ of $\Re$ to be the semantic value of either a simple or a complex predicate. That subset is indeed an open subset of $\mathfrak{R}$ under the usual topology. One might reasonably doubt, however, whether a set such as this can really serve as the semantic value of a vague predicate. Can it really be that the wavelengths of red light, for example, are those lying 
in a certain range of a real numbers with the sole exception of a single real number lying within that range?

The natural answer is no: our understanding of the term 'red' seems to preclude such a set as an admissible extension of the term. It is, however, vital that such sets should be admissible extensions of predicates if Tarski's proposition $\left(B^{\prime}\right)$ is to hold.

Indeed, the case suggests a stronger condition for a set to serve as the semantic value of a vague predicate. Topologists call a set regular open if it is the interior of its own closure. Any regular open set is open, but $(0, \infty) \cup(-\infty, 0)$ is an example of a set which is open without being regular. The closure of $(0, \infty) \cup(-\infty, 0)$ is $\Re$ itself, which is also the interior of the closure. Regular open sets, then, have no 'cracks' in them, and one might postulate that any admissible extension of a vague term will be regular open, not merely open. Given appropriate definitions of meet, join, and complement, however, the regular open subsets of any topological space form a Boolean algebra. $1^{12} /$ If the extensions of vague predicates are required to be regular open, and 'and', 'or' and 'not' are interpreted using the appropriate definitions of meet, join, and complement, then the semantics validates the full classical logic. The stronger topological requirement, then, refutes Wright's hypothesis that intuitionistic logic is the logic of vagueness and therewith his solution to the Sorites.

That stronger requirement, though, opens the way to an alternative solution to the ancient puzzle. As the case of $(0, \infty) \cup(-\infty, 0)$ shows, the union of two regular open sets need not be regular open. Accordingly, the join operation of the Boolean algebra of regular open sets is not $X \cup Y$, but $\operatorname{Int} C l(X \cup Y)$. If the Boolean algebra is to validate classical logic, it is this join operation that must be assigned to interpret the predicate connective 'or' and hence the sentential connective ' $\vee$ '. Under this interpretation, ' $A \vee B$ ' may be true even though neither $A$ nor $B$ is true. Now Wright's 'Unpalatable Existential' (10), viz.,

(10) $\quad \exists n\left(a_{n}\right.$ is red $\wedge \neg\left(a_{n+1}\right.$ is red $\left.)\right)$,

\footnotetext{
12 This result follows directly from the Stone Representation Theorem (Stone 1936). Although Stone was the first to publish the result, Tarski liked to claim credit for it: 'This fact was noticed by me as far back as 1927, and was implicitly stated in Theorem B of [Tarski 1929], where, however, a different terminology was used' (Tarski 1938, 449). The interested reader may like to ponder in what sense the result in 'implicit' in Theorem B of Tarski 1929; see Tarski 1983, 29.
} 
is no more than the following long disjunction

$\left(10^{\prime}\right) \quad\left(a_{1}\right.$ is red $\wedge \neg\left(a_{2}\right.$ is red $\left.)\right) \vee \ldots \vee\left(a_{99}\right.$ is red $\wedge \neg\left(a_{100}\right.$ is red $\left.)\right)$.

On the alternative semantics now proposed, then, $\left(10^{\prime}\right)$ may be true even though no disjunct ' $a_{n}$ is red $\wedge \neg\left(a_{n+1}\right.$ is red $)$ ' is true. Wright solves the Sorites by revising classical logic so that we do not reach $(10)$ or $\left(10^{\prime}\right)$. On the alternative approach, the inference to $(10)$ or $\left(10^{\prime}\right)$ need not be resisted. For, under the alternative interpretation, Wright's 'Unpalatable Existential' is not really unpalatable: it does not ascribe a sharp boundary to the red members of the Sorites sequence.

I developed this solution to the Sorites in Chapter 8 of The Boundary Stones of Thought (see Rumfitt 2015, 250f.). I argued there that the extension of a vague predicate will be regular open whenever the predicate is 'polar' in Mark Sainsbury's sense - that is, when its meaning is given by reference to a system of contrary paradigms or poles (see Sainsbury 1990). I stand by that argument. However, I never pretended to have an argument for the conclusion that every vague predicate is polar. So I have no argument for the thesis that every vague predicate has a regular open extension.

For this reason, it seems to me to be well worth exploring further the semantic theory sketched in $\S \S 3-4$. It seems likely that the semantic values of some families of vague predicates must be regular open, not merely open. When reasoning with these predicates, we would appear to be entitled to apply all the rules of classical logic, so we shall need a solution to the Sorites different from Wright's. There is, though, no reason I can discern to hold that all vague predicates must have regular open sets as their semantic values. Perhaps having an open set as its semantic value is the strongest general requirement that any vague predicate must satisfy. If the topological space in which the set is open is Euclidean, then this requirement will sustain intuitionistic logic. That logic, then, may be the strongest logic that we are entitled to use in reasoning with any vague predicate. Perhaps we are entitled to use the classical logic only when reasoning with certain families - albeit central and common families — of vague term. As the many 
occurrences of 'may be' and 'perhaps' in this paragraph show, I do not know the answers to these questions. I hope, though, to have indicated some promising directions for further inquiry. $1^{13} /$

\footnotetext{
13 I first found myself entertaining these ideas while scribbling on whiteboards during tutorials on vagueness given to finalists and B.Phil. students at Oxford; I hope that on this occasion the ideas are at least legible. Drafts of the paper have provided the basis for seminars and lectures at Oxford and London, and for talks at the Universities of Bristol and Leeds and at the Moral Sciences Club in Cambridge. I thank members of all my audiences for helpful questions and remarks. I am particularly indebted to Timothy Williamson for written comments, and to Mark Sainsbury for his advice and encouragement.
} 


\section{REFERENCES}

Auxier, R.E. and L.E. Hahn, eds. 2007. The Library of Living Philosophers: Michael Dummett.

Chicago, Ill.: Open Court.

Dummett. M.A.E. 1975. 'Wang’s Paradox'. Synthèse 30: 301-24.

— 1991. The Logical Basis of Metaphysics. London: Duckworth.

— 2007. 'Reply to Crispin Wright'. In Auxier and Hahn, eds., pp.445-54.

Frege, G. 1896. 'Letter to Peano, 29 September 1896'. In Frege 1976, pp.181-86. English translation in Frege 1980, pp.112-18.

—_ 1897-8. 'Begründung meiner strengeren Grundsätze des Definierens'. In Frege 1969, pp.164-170. English translation in Frege 1979, pp.152-56.

— . 1902. Grundgesetze der Arithmetik, volume II. Jena: Hermann Pohle.

— 1969. Nachgelassene Schriften, eds. H. Hermes et al. Hamburg: Felix Meiner.

— . 1976. Wissenschaftlicher Briefwechsel, eds., G. Gabriel et al. Hamburg: Felix Meiner.

—. 1979. Posthumous Writings, trans. P. Long and R. White. Oxford: Blackwell.

_ 1980. Philosophical and Mathematical Correspondence, trans. H. Kaal. Oxford: Blackwell.

Putnam, H.W. 1983. 'Vagueness and Alternative Logic'. In his Realism and Reason: Philosophical Papers Volume 3 (Cambridge: Cambridge University Press), pp.271-286.

—. 1985. 'A Quick Read is a Wrong Wright'. Analysis 45: 203.

Read, S.L. and C.J.G. Wright. 1985. 'Hairier than Putnam Thought'. Analysis 45: 56-8.

Rumfitt, I. 2015. The Boundary Stones of Thought: An Essay in the Philosophy of Logic. Oxford: Clarendon Press. 
Sainsbury, R.M. 1990. Concepts without Boundaries. London: King's College. Reprinted in Keefe and Smith, eds., Vagueness: A Reader (Cambridge, Mass.: MIT Press, 1996), pp.251-64.

Stone, M.H. 1936. 'The Theory of Representations for Boolean Algebras'. Transactions of the American Mathematical Society 11: 37-111.

Tarski, A. 1929. 'Les Fondements de la Géometrie des Corps'. Annales de la Société Polonaise de Mathématique, supplement, pp.29-33. Page references are to the translation by J.H. Woodger, entitled 'Foundations of the Geometry of Solids', in Tarski 1983, pp.24-9.

—. 1935. 'Der Wahrheitsbegriff in den formalisierten Sprachen'. Studia Philosophica 1: 261-405.

Page references are to the translation by Woodger, entitled 'The Concept of Truth in Formalized Languages', in Tarski 1983, pp.152-278.

—_ 1938. 'Der Aussagenkalkül und die Topologie'. Fundamenta Mathematicae 31: 103-34. Page references are to the translation by Woodger, entitled 'Sentential Calculus and Topology', in Tarski 1983, pp.421-54.

— 1983. Logic, Semantics, Metamathematics: Papers from 1923 to $1938,2^{\text {nd }}$ edition, eds. J.H.

Woodger and J. Corcoran. Indianapolis: Hackett.

Williamson, T. 1994. Vagueness. London: Routledge.

Wright, C.J.G. 1975. 'On the Coherence of Vague Predicates'. Synthèse 30: 325-65.

—. 2001. 'On Being in a Quandary: Relativism, Vagueness, Logical Revisionism'. Mind 110: 45-98.

—. 2003. 'Rosenkranz on Quandary, Vagueness and Intuitionism'. Mind 112: 465-74.

—. 2007. “"Wang's Paradox”. In Auxier and Hahn, eds., pp.415-44. 